\title{
La bradykinine
}

\section{Jean-Loup Bascands Jean-Pierre Girolami}

\section{ADRESSE}

J.L. Bascands : ingénieur de recherche à l'Inserm; J.P. Girolami : directeur de recherche à l'Inserm. Inserm U. 388, Institut Louis Bugnard, 1, avenue J.-Poulhes, CHU Rangueil, 31054

Initialement connue pour ses propriétés vasodilatatrices, la bradykinine a de multiples effets : contraction et/ou relaxation des muscles lisses, libération de neuromédiateurs, stimulation de la prolifération cellulaire. Ces effets sont relayés par deux types de récepteurs: B2, trouvé dans un grand nombre de tissus, et B1, induit le plus souvent dans des états pathologiques. Les voies de transduction du signal bradykinine mettent en jeu les différents seconds messagers connus $\left(\mathrm{Ca}^{2+}\right.$, prostaglandines, $\left.\mathrm{NO}, \mathrm{GMPc}\right)$. Un excès de bradykinine peut être impliqué dans des réactions inflammatoires (allergie, asthme, oedème) et, dans ce cas, le blocage des récepteurs par des antagonistes spécifiques aurait des effets bénéfiques. Inversement, une diminution de l'activation des récepteurs au niveau vasculaire pourrait être associée à l'aggravation du tonus vasoconstricteur et au développement d'anomalies vasculaires. L'activation du récepteur B2 pourrait être impliquée dans le mécanisme d'action de médicaments antihypertenseurs tels que les inhibiteurs de l'enzyme de conversion de l'angiotensine II.

es kinines sont des peptides naturels dont le plus connu est le nonapeptide bradykinine (BK). Les kinines résultent de l'hydrolyse de deux substrats (kininogènes) par au moins deux protéases à sérine, les kallicréines plasmatique et tissulaire (figure 1). Les kinines sont métabolisées par de nombreuses kininases, dont la plus connue, la kininase II, n'est autre que l'enzyme de conversion de l'angiotensine; son inhibition a donné naissance à une famille de médicaments antihypertenseurs: les inhibiteurs de l'enzyme de conversion (IEC).

\section{Déjà une longue} et vieille histoire

L'histoire de ce système commence dès 1909 quand Abelous et Bardier, deux physiologistes toulousains, rapportèrent que l'injection intraveineuse d'urine humaine entraînait, chez le chien, une importante chute de la pression sanguine systolique [1]. Ces chercheurs avaient, sans le savoir, découvert l'effet de la kallicréine. Sur la base de l'observation des deux physiologistes, le groupe de E. Werle du Max Plank Institüt de Munich isola, en 1930, des quantités importantes de cette substance hypotensive 
à partir d'extraits de pancréas, la désigna du nom de «kallicréine» (du grec kallikreas, pancréas) [2] et suggéra qu'il s'agissait d'une hormone synthétisée par le pancréas et éliminée dans l'urine, hypothèse abandonnée avec la découverte des enzymes plasmatiques et tissulaires. En 1950, le groupe brésilien de Rocha e Silva [3] découvrit la BK en montrant que la trypsine ou le venin du serpent Bothrops jararaca, mis en présence de globulines plasmatiques, induisait la libération d'un peptide fortement hypotenseur. Ces chercheurs attribuèrent les noms de "bradykinine» à ce peptide, de «bradykininogène » à son précurseur et de "kininogénases" aux enzymes telles que la kallicréine, la trypsine, l'enzyme du venin de serpent, les protéases bactériennes, etc., qui sont capables de libérer des kinines à partir du précurseur. En 1970, aux ÉtatsUnis, le groupe d'Erdös découvrit que l'enzyme de conversion de l'angiotensine est à la fois capable de produire de l'angiotensine II vasoconstrictrice et d'inactiver la BK vasodilatatrice [4]. Cette découverte ouvrit alors une importante voie de recherche pour l'industrie pharmaceutique dans le domaine de l'hypertension avec le développement des IEC. Après plusieurs années de recherches sur la structure de la BK, Vavreck et Stewart de l'École de Médecine de Denver synthétisaient le premier antagoniste compétitif de la BK [5]. En 1980, D. Regoli et J. Barabe [6] proposaient, sur des bases exclusivement pharmacologiques, l'existence de deux récepteurs de la BK. Cette distinction fut confirmée en 1991 et 1994 par le clonage des ADNc de deux types de récepteurs distincts. L'histoire de la bradykinine s'accélèra tout en restant encore largement mystérieuse. Les données ponctuelles se sont accumulées mais les grandes questions subsistent: quels sont les rôles physiologiques et pathologiques pour la BK? effets bénéfiques ou effets délétères?

\section{Biologie de la bradykinine}

\section{Biosynthèse et dégradation}

Pendant de nombreuses années on a distingué deux systèmes kinine-kallicréine: un plasmatique et un tissulai- re. En fait, n'existent que deux enzymes kallicréines qui présentent des localisations, des propriétés physico-chimiques et fonctionnelles bien distinctes [7]. Les kallicréines sont des protéases à sérine parmi lesquelles on distingue la kallicréine plasmatique (MM: $60 \mathrm{kDa}$ ), synthétisée par le foie, des kallicréines tissulaires (MM entre 24 et $48 \mathrm{kDa}$ ) qui sont principalement synthétisées par le pancréas, le rein, l'intestin, le cerveau, les glandes sousmaxillaires et sudoripares. La kallicréine plasmatique est codée par un seul gène, alors que les kallicréines tissulaires sont codées par plusieurs gènes, localisés chez l'homme sur le chromosome 19, appartiennent à une famille multigénique [8]. Toutes deux hydrolysent avec des affinités différentes le kininogène de haut et de bas poids moléculaire (figure 1). Les kininogènes de haut et de bas poids moléculaire synthétisés dans le foie proviennent de l'épissage alternatif d'un seul gène [7] et présentent des variations de séquence en fonction des espèces. En conséquence, on observe également des variations interspécifiques dans la séquence peptidique des kinines.

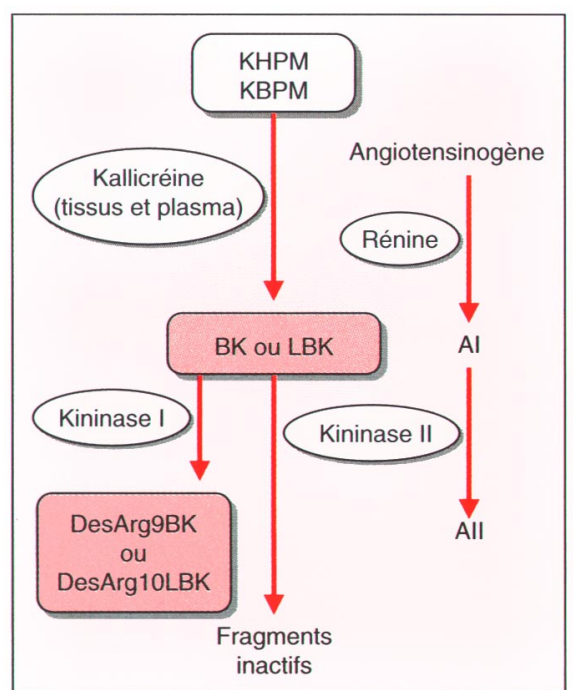

Figure 1. Organisation des systèmes kinines kallicréine: formation et dégradation des kinines, parallélisme avec le système rénine-angiotensine. KHPM: kininogène de haut poids moléculaire; KBPM : kininogène de bas poids moléculaire; $B K$ : bradykinine; Lys-Bk: lysyl-bradykinine; Al: angiotensine I; All: angiotensine II; Kininase I: carboxypeptidase $N$ et $M$; Kininase II: enzyme de conversion.
Chez l'homme et le bœuf, existent trois kinines principales, la kallidine ou lys-bradykinine (lys-BK) qui est un décapeptide natif issu de l'action de la kallicréine, la bradykinine (BK) qui est un nonapeptide (Arg-Pro-ProGly-Phe-Ser-Pro-Phe-Arg) et l'octapeptide des-Arg ${ }^{9}$-BK. Chez le rat, on trouve uniquement de la BK et de la dés-Arg'-BK ainsi qu'une kinine spécifique du rat, Ile-Ser-BK, encore appelé T-kinine [7]. Cette kinine spécifique est libérée à partir d'un kininogène, le T-kininogène, identifié uniquement chez le rat et hydrolysable uniquement par la trypsine. Ce T-kininogène n'apparaît pour l'instant que comme un marqueur de l'inflammation [9]. Plus récemment, des variants de la lys-BK et de la BK ont été mis en évidence, dans lesquels la proline en position 3 est hydroxylée [7]. Ces variants existent chez l'homme et les primates mais pas chez les rongeurs ni les bovins. Ces modifications proviendraient de modifications post-traductionnelles du kininogène.

La demi-vie plasmatique des kinines est estimée à environ 15 secondes. Les principales enzymes (figure 1) dégradant les kinines sont la kininase I, également connue sous le nom de carboxypeptidase N (CPN dans le plasma) ou encore carboxypeptidase M (CPM sur la membrane plasmique des cellules vasculaires). Ces enzymes engendrent la dés-Arg' ${ }^{9}$-BK qui est un peptide biologiquement actif avec des effets biologiques bien distincts de ceux de la BK. La kininase II, plus connue sous le nom d'enzyme de conversion de l'angiotensine II, dégrade les kinines en fragments BK1-5 et BK1-7 inactifs, et assure également la conversion de l'angiotensine I en angiotensine II (figure 1). Cette enzyme de conversion a une plus grande affinité pour la BK que pour l'angiotensine I [10].

Les bradykinines auraient une action locale

La rapidité d'inactivation des kinines par les kininases, laisse supposer que les kinines présentes dans la circulation ne sont pas impliquées dans l'activation des récepteurs. Ainsi s'est dessiné un concept d'action locale de type autocrine ou paracrine. $\mathrm{Au}$ niveau vasculaire, un ensemble de 
travaux suggère que la cellule endothéliale serait capable de produire de la BK $[7,11]$. Cette hypothèse implique que la cellule endothéliale possède la machinerie nécessaire à la production de BK. Quelques travaux indiquent l'existence d'une activité kallicréine dans la paroi vasculaire $[7,11]$. La présence de kininogène reste plus hypothétique. En effet, malgré la disponibilité des techniques de biologie moléculaire, l'existence d'un système kallicréine intrinsèque à la paroi vasculaire reste à démontrer de façon irréfutable. En revanche, récemment des sites de fixation des kininogènes ont été mis en évidence, suggérant que la cellule endothéliale pourrait capter le substrat circulant et ainsi engendrer de la BK [12].

\section{Hétérogénéité des récepteurs des kinines}

3. Rocha e Silva M, Beraldo W, Rosenfeld G. Bradykinin, a hypotensive and smooth muscle stimulating factor released from plasma globulin by snake venoms and by trypsin. Am J Physiol 1949; 156 : 261-73.

4. Yang HYT, Erdös EG, Levin Y. A dipeptidyl carboxypeptidase that converts angiotensin I and inactivates bradykinin. Biochim Biophys Acta 1970; 214: 374-6.

5. Vavreck RJ, Stewart JM. Competitive antagonists of bradykinin. Peptide $1985 ; 6$ : 161-4.

6. Regoli D, Barabe J. Pharmacology of bradykinin and related kinins. Pharmacol Rev 1980; 32: 1-46.

7. Bhoola KD, Figueroa CD, Worthy K. Bioregulation of kinins : kallikreins, kininogens and kininases. Pharmacol Rev 1992; 44: 1-80.

8. Clements JA. The glandular kallikrein family of enzymes: tissue-specific expression and hormonal regulation. Endocrine Rev $1989 ; 10$ : 393-419.

9. Gauthier F, Gutman N, Moreau T, Elmoujahed A. Possible relationship between the restricted biological function of rat T-kininogen (thiostatin) and its behaviour as an acute phase reactant. Hoppe-Seylers $Z$ Biol Chem 1988; 369 : 251-5.

10. Jaspard E, Wei L, Alhenc-Gélas F. Differences in the properties and enzymatic specificities of the two active sites of angiotensin I-converting enzyme (kininase II). J Biol Chem 1993; 268 : 9496-503.

11. Corvol P, Alhenc-Gélas F, Soubrier F. L'endothélium, site de production et de métabolisme des peptides vaso-actifs. médecine/sciences 1993; 9: 1050-61.

12. Hasan AAK, Cines DB, Zhang J, Schmaier AH. The carboxyl terminus of bradykinin and amino terminus of the light chain of kininogens comprise an endothelial cell binding domain. J Biol Chem 1994;
A la suite de nombreuses études des relations entre les modifications de la séquence peptidique de la BK et son activité biologique, une première classification des récepteurs de la BK a été proposée par Regoli et Barabe en 1980 [6]. Étudiant l'effet de la BK et de la dés-Arg'-BK sur la contraction de différentes préparations vasculaires, ces auteurs ont distingué deux types de récepteurs nommés $B_{1}$ et $B_{2}$ Le récepteur $B_{1}$, d'abord identifié sur l'aorte et la veine mésentérique de lapin, est activé préférentiellement par la dés-Arg ${ }^{9}$-BK mais également par la BK avec une moindre efficacité. Le récepteur B2, présent sur la veine jugulaire dé lapin et la carotide de chien, est stimulé exclusivement par la BK et la Lys-BK.

Par ailleurs, différentes substitutions dans la séquence peptidique de la BK ont permis d'identifier des positions importantes. Ainsi la suppression du résidu arginine en position 9 (dés$\mathrm{Arg}^{9}$-BK), transforme la BK, agoniste préférentiel de type B2, en agoniste sélectif de type B1. La substitution $\mathrm{Pro}^{7}$ en $\mathrm{Phe}^{7}$ transforme la molécule de BK en antagoniste de type B2 alors que la substitution $\mathrm{Phe}^{8}$ en $\mathrm{Leu}^{8}$ transforme la dés-Arg ${ }^{9}-\mathrm{BK}$, agoniste $\mathrm{B} 1$, en antagoniste B1. Les antagonistes les plus remarquables sont mentionnés sur le Tableau I. Les antagonistes peptidiques de première génération n'avaient, comparés à la BK elle-même, qu'une faible affinité pour le récepteur B2. La deuxième génération d'antagonistes est caractérisée par l'introduction de résidus de chaînes aliphatiques en position 7 , ce qui a considérablement augmenté leur résistance à la dégradation. Le plus connu de ces antagonistes est sans aucun doute le HOE 140, dépourvu d'affinité pour le récepteur B1. Les composés NPC17731 et CP0127 sont des homodimères qui présentent quasiment les mêmes caractéristiques que le HOE 140. Plus récemment, un hétérodimère a été développé (CP0364), qui contient à la fois un antagoniste B2 et un antagoniste B1 [13]. Enfin, la troisième génération très récente d'antagonistes est celle des molécules non peptidiques. Le WIN64338 représente le premier antagoniste non peptidique du récepteur B2 [13]. Cet antagoniste présente une assez bonne affinité pour le récepteur B2 de l'iléon de cobaye et pour les cellules fibroblastiques de poumons humains IMR-90 [13]. Initialement présenté comme inactif sur le récepteur B1, Wirth et al. [14] ont rapporté récemment que cet antagoniste inhibe l'augmentation de production du GMPc intracellulaire provoquée par la dés-Arg ${ }^{9}$-BK sur les cellules endothéliales d'aorte de bouf.

Le récepteur B2, qui a été le plus étudié, est présent dans un grand nombre de tissus en quantité plus élevée que le récepteur B1. Cependant, de nombreuses études pharmacologiques, utilisant des agonistes et des antagonistes suggèrent l'existence d'une population hétérogène de récepteurs de type B2. Différentes hypothèses sont avancées: d'une part, l'existence de sous-populations de récepteurs différant par le niveau de couplage avec les protéines $G$ et incluant même une population de récepteurs constitutivement actifs; d'autre part, des populations différant par le nombre et la nature des protéines G couplées [15]. Plus récemment, Regoli et al. [16] ont distingué deux sous-types du récepteur $\mathrm{B} 2$ : le récepteur $\mathrm{B} 2_{\mathrm{rb}}$ ou $\mathrm{B} 2_{\mathrm{A}}$ (veine jugulaire de lapin) et $\mathrm{B} 2_{\mathrm{gp}}$ ou $\mathrm{B} 2_{\mathrm{B}}$ (iléon de cobaye), indiquant que l'hétérogénéité des récepteurs B2 semble plus associée à des différences d'espèces qu'à une réelle hétérogé- 


\begin{tabular}{|lll|}
\hline \multicolumn{2}{c|}{ Tableau I } \\
& \multicolumn{1}{c|}{ ANTAGONISTES DES RÉCEPTEURS B1 ET B2 } \\
\hline Antagonistes & B1 & B2 \\
\hline $1^{\text {re }}$ génération & [Leu8-des Arg9]BK & [DPhe7]-BK \\
& [Leu9-des Arg10]Lys-BK & [DArg-Hyp3-Thi5,8-DPhe7]-BK \\
$2^{\mathrm{e}}$ génération & DArg[Hyp3,Thi5,Cys6,DPhe7]BK & DArg[Hyp3,Thi5,Cys6,DPhe7]BK \\
& S-linker-S & S-linker-S \\
& DArg[Cys1,Hyp3,Thi5,DPhe7 & DArg[Cys1,Hyp3,Thi5,DPhe7 Leu8] (a) \\
& Leu8] (a) & DArg[Hyp3,Thi5,DTic Oic8]BK (b) \\
& & DArg[Hyp3,DHyp7,Oic8]BK (c) \\
& & DArg[Hyp3,Thi5,Cys6,DPhe7]2-BK (d) \\
$3^{3}$ génération & & WIN 64338 (e) \\
& & \\
\hline
\end{tabular}

(a): CP-0364; (b): HOE140; (c): NPC1773; (d): CP-0127; (e) : [[4[[2[[bis(cyclohexylamino)méthylène]amino]-3-(2naphthyl-1oxopropyl]amino]phenyl]phenyl]trietylphosphonium chloride monohydrochloride.

néité moléculaire. Enfin, certains auteurs ont même proposé, uniquement sur la base des effets d'antagonistes, l'existence de récepteurs $\mathrm{B} 3$, B4 et B5 [13], qui n'a pas été confirmée à l'échelle moléculaire.

Pendant de nombreuses années le récepteur B1 est apparu comme une curiosité d'espèce n'ayant été étudié que sur l'aorte de lapin. Le développement méthodologique a permis sa mise en évidence, dans d'autres tissus et dans d'autres espèces, notamment chez l'homme [15]. Il est aussi synthétisé dans différents types cellulaires en culture où il entraîne la mobilisation du calcium intracellulaire et stimule la prolifération [15, 17]. En comparant les effets de la BK et de la dés-Arg'-BK sur le calcium libre intracellulaire, nous avons pu mettre en évidence la coexistence des deux types de récepteurs B1 et B2 sur la cellule mésangiale de rat [17]. Par la suite, la coexistence des récepteurs $\mathrm{B} 1$ et $\mathrm{B} 2$ a été démontrée dans plusieurs autres types cellulaires.

L'utilisation des ligands radioactifs, en général des agonistes tritiés ou iodés, a permis de distinguer et de quantifier les deux types de récepteurs B1 et B2. De nombreux travaux ont été consacrés à la détection du site de liaison B2 par des tests de liaison [18]. Malgré leur courte demivie, les ligands iodés présentent l'avantage d'avoir une meilleure radioactivité spécifique comparés aux ligands tritiés. Bien que cela modifie la molécule native, la présence d'un résidu tyrosine sur les ligands iodés n'altère pas leur efficacité biologique. Quelques études ont été réalisées avec $\left.{ }^{[125} \mathrm{I}\right]-\mathrm{Tyr}^{0}-\mathrm{BK}$ [19] qui apparaît comme un radioligand biologiquement actif plus efficace que [ $\left.{ }^{125} \mathrm{I}\right]-\mathrm{Tyr}^{8}-\mathrm{BK}$. Pour plus d'informations, un tableau détaillé des sites de liaison B2 de la BK dans divers tissus de différentes espèces est présenté dans la revue de Hall [18]. Si la plupart des travaux montrent un seul site de liaison, quelques-uns rapportent l'existence d'un deuxième site de liaison de très forte affinité $[17,18]$.

Les premiers travaux réalisés sur le récepteur $\mathrm{B} 1$ ont montré une liaison saturable de la $\left[{ }^{3} \mathrm{H}\right]$ dés-Arg. ${ }^{9}$-BK sur la veine mésentérique de lapin, qui augmente après un prétraitement de quelques heures par le lipopolysaccharide, ce qui est en plein accord avec le caractère inductible du récepteur B1 [15]. Plus récemment, un radioligand spécifique du récepteur $\mathrm{B} 1$, la $\left[{ }^{3} \mathrm{H}\right]$-dés-Arg ${ }^{10}$-kallidine, a permis de caractériser ce récepteur sur la lignée de macrophages RAW2647; son affinité (Kd) est de 2,4 nM et sa densité (Bmax) de 700 sites/cellule [15]. Avec le même radioligand nous avons pu mettre en évidence, chez le rat, la présence du récepteur B1 sur le glomérule isolé de rat et sur les cellules mésangiales rénales en culture, présentant un Kd de 8,7 nM et un Bmax d'environ 2000 sites/cellule [17].

\section{Approche moléculaire}

De façon tout à fait remarquable, le clonage des $\mathrm{ADNc}$ et des gènes des récepteurs de la $\mathrm{BK}$ a confirmé, près de 15 ans plus tard, leur classification pharmacologique. Ainsi, les ADNc codant pour le récepteur B2 du rat [20] et de l'homme [21] ont été isolés. L'ADNc codant pour le récepteur B1 de l'homme a été décrit plus récemment [22]. Ces récepteurs appartiennent à la famille des récepteurs à sept domaines transmembranaires couplés aux protéines G. Les récepteurs B2 du rat et de l'homme sont composés respectivement de 366 et 364 acides aminés. Il y a environ $81 \%$ d'identité. Chacun d'eux présente trois sites de $\mathrm{N}$-glycosylation sur les domaines extracellulaires et quatre sites potentiels de phosphorylation (deux pour la protéine kinase A et deux pour la protéine kinase $C$ ) dans les domaines intracellulaires. Powell et al. [23] ont localisé le gène du récepteur B2 humain sur le chromosome 14. Chez l'homme, le récepteur B1 est composé de 353 acides aminés et présente $36 \%$ d'identité avec le récepteur B2 (figure 2). Le récepteur $\mathrm{B} 1$ possède trois sites de $\mathrm{N}$ glycosylation, quatre sites potentiels de phosphorylation par la protéine kinase C (PKC) et un site de phosphorylation par la protéine kinase A. Récemment, Pesquero et al. [24] ont décrit précisément la structure du gène du récepteur $B 2$ chez le rat. Le gène de ce récepteur serait constitué 


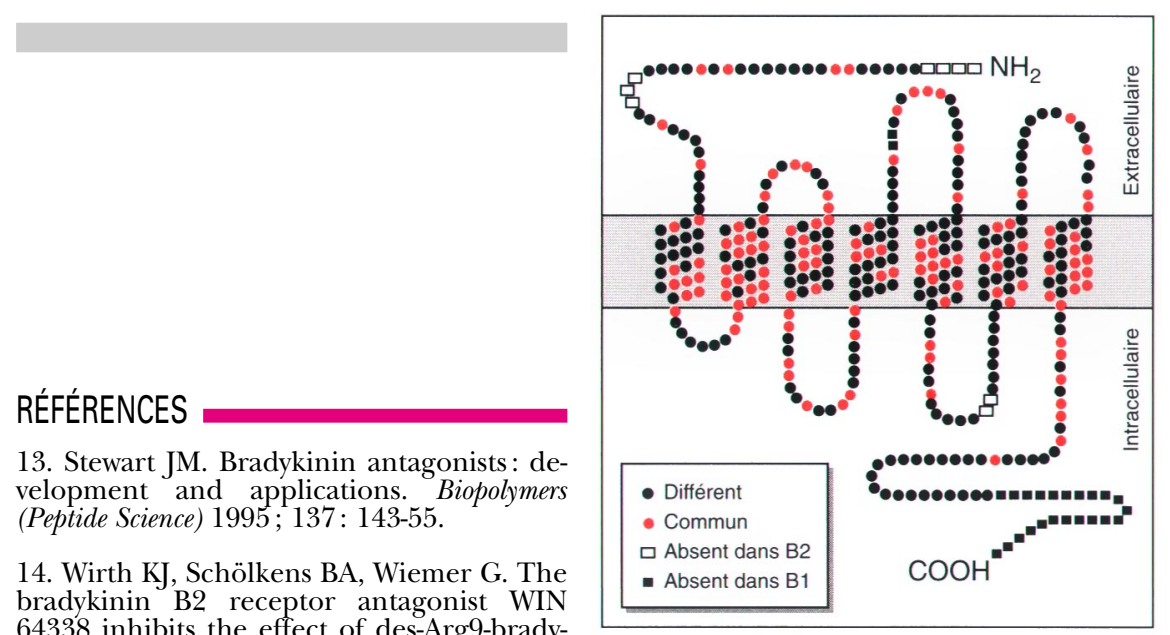

64338 inhibits the effect of des-Arg9-bradykinin in endothelial cells. Eur I Pharmacol 1994; 228: R1-2.

15. Burch RM, Kyle DJ, Storman N. Molecular biology and pharmacology of bradykinin receptors. In: Molecular biology intelligence unit. Austin: RG Landes Company, 1995: 107.

16. Regoli D, Gobeil F, Nguyen QT. Bradykinin receptor types and $\mathrm{B}_{2}$ subtype. Life Sci $1994 ; 50: 735-49$.

17. Bascands JL, Pecher C, Rouaud S, Emond C, Leung-Tack J, Bastie MJ, Burch R, Regoli D, Girolami JP. Evidence for existence of two distinct bradykinin receptors on rat mesangial cells. Am J Physiol 1993; 264: F548-56.

18. Hall JM. Bradykinin receptors : pharmacological properties and biological roles. Pharmacol Ther 1992; 56: 131-90.

19. Emond C, Bascands JL, Pecher C, Cabos-Boutot G, Pradelles P, Regoli D, Girolami JP. Characterization of a $B_{2}$ bradykinin receptor in rat renal mesangial cells. Eur J Pharmacol 1990; 190 : 381-92.

20. McEachern AE, Shelton ER, Bhakta S, Obernolte R, Bach C, Zuppan P, Fujisaki J, Aldrich RW, Jarnagin K. Expression cloning of a rat $\mathrm{B}_{3}$ bradykinin receptor. Proc Natl Acad Sci USA 1991 ; 88: 7724-8.

21. Hess JF, Borkowski JA, Young GS, Strader CD, Ransom RW. Cloning and pharmacological characterization of a human bradykinin (BK-2) receptor. Biochem Biophys Res Commun 1992; 184: 260-8.

22. Menke JG, Borkowski JA, Bierilo KK, Mac Neil T, Derrick AW, Schneck KA, Ransom RW, Strader CD, Linemeyer DL, Hess JF. Expression cloning of a human B1 bradykinin receptor. I Biol Chem 1994; 269: 21583-6.

23. Powell SJ, Slynn G, Thomas C, Hopkins B, Briggs I, Graham A. Human bradykinin B2 receptor: nucleotide sequence analysis and assignment to chromosome 14. Genomics 1992 ; 15 : 435-8.

24. Pesquero JB, Lindsey CJ, Zeh K, Paiva ACM, Ganten D, Bader M. Molecular structure and expression of rat bradykinin $\mathrm{B} 2$ re-
Figure 2. Comparaison de la séquence en acides aminés des récepteurs B1 et B2 chez l'homme.

de quatre exons interrompus par trois introns. La région codante est entièrement localisée sur le quatrième exon. La région promotrice reste encore mal connue. Il est intéressant de noter que l'on ne trouve pas de séquences CAAT ou TATA. Cette les gènes codant pour les récepteurs couplés aux protéines $G$ tels que les récepteurs A1 de l'adénosine, de la dopamine, des endothélines, etc. On trouve une région 3' non traduite relativement longue contenant deux motifs AUUUA qui joueraient un rôle dans l'instabilité des ARN messagers. Les expériences de mutagenèse dirigée ont permis de déterminer les acides aminés impliqués dans la reconnaissance des agonistes et des antagonistes [15]. Ces travaux ont principalement montré que le site de liaison de la bradykinine est différent des sites de liaison des antagonistes. Les études de la distribution de l'ARNm codant pour le récepteur B2 réalisées par la technique de Northern blot ont montré, tant chez le rat que chez l'homme, une forte expression dans le rein, l'utérus et le poumon et une expression plus faible dans le cerveau, le cœur, les testicules et le pancréas. La création récente [25] d'une souche de souris transgéniques n'exprimant plus le récepteur B2 confirme l'existence d'un seul gène codant pour ce récepteur. La BK pouvant activer les deux types de récepteurs B1 et B2, ces animaux absence semble assez fréquente pour transgéniques seront un excellent modèle d'étude des effets physiologiques du récepteur $\mathrm{B} 1$.

\section{Les seconds messagers de la bradykinine}

Alors que de nombreux travaux montrent finalement que la BK stimule la totalité des voies de signalisation connues dans l'ensemble des types cellulaires étudiés [15, 18], la nature des protéines $\mathrm{G}$ impliquées est moins bien connue. Dans la majorité des cas, l'activation de la phospholipase C (PLC) par la BK est insensible au traitement par la toxine pertussique alors que l'activation de la phospholipase A2 (PLA2) est, au contraire, le plus souvent inhibée indiquant un couplage du récepteur B2 à une protéine de type Gi. Cependant, la nature des protéines $G$ couplées aux récepteurs de la BK n'a été clairement identifiée que dans deux études. Ces travaux révèlent un couplage avec une protéine $\mathrm{G}$ de type G $\alpha q$ dans les cellules NG108-15 et $\mathrm{G} \alpha \mathrm{q}$ et $\mathrm{G} \alpha_{\mathrm{i}}$ dans les cellules endothéliales d'aorte de bœuf [26]. Le Tableau II résume les différentes voies de transduction activées par la BK dans différents types cellulaires.

La stimulation du récepteur B2, dans presque tous les types cellulaires étudiés (cellules épithéliales, fibroblastes, cellules de neuroblastomes, cellules mésangiales, cellules musculaires lisses), conduit à l'activation de la voie de la PLC suivie de la formation d'inositol phosphate et de diacylglycérol. La BK entraîne une libération biphasique du $\left[\mathrm{Ca}^{2+}\right] \mathrm{i}$ : une phase rapide et transitoire provenant de la libération du $\mathrm{Ca}^{2+}$ du réticulum endoplasmique, et une phase plus lente et soutenue, associée à l'activation de canaux calciques. Tant sur des fibroblastes [15] que sur les cellules mésangiales [17] la phase soutenue de la mobilisation du calcium est inhibée par le chlorure de nickel (bloqueur des canaux calciques de type T) alors que les bloqueurs de canaux de type L (nifédipine, vérapamil) sont sans effets. Dans presque tous les tissus la BK active la PLA2 engendrant ainsi de l'acide arachidonique qui est métabolisé en eicosanoïdes. Ces derniers varient en fonction du type cellulaire étudié; on trouve principalement les prostaglandines $\mathrm{E}_{2}$ et 
Tableau II

VOIES DE TRANSDUCTION DE LA BRADYKININE DANS DIVERS TISSUS

\begin{tabular}{|c|c|c|c|}
\hline Tissus/type cellulaire & Récepteurs & Protéines G/Effecteurs & Seconds messagers/effets \\
\hline $\begin{array}{l}\text { Fibroblastique } \\
\text { - Swiss 3T3 (souris) } \\
\text { - IMR-90 (homme) }\end{array}$ & $\begin{array}{l}\text { B2 } \\
\text { B1-B2 }\end{array}$ & $\begin{array}{l}\text { PLC/PLA2 } \\
\text { PLC/PLA2 }\end{array}$ & $\begin{array}{l}\left.\uparrow \text { IP3, [Ca }{ }^{2+}\right] \text { i, PGE2 } \uparrow\left[\mathrm{Ca}^{2+}\right] \mathrm{i} \text {, } \\
\text { PG.Prolifération }\end{array}$ \\
\hline $\begin{array}{l}\text { Épithélial } \\
\text { - rein (lapin) } \\
\text { - tube collecteur (lapin) } \\
\text { - MDCK (chien) }\end{array}$ & $\begin{array}{l}\text { B2 } \\
\text { B2 } \\
\text { B2 }\end{array}$ & $\begin{array}{l}\text { PLC/PLA2 } \\
\text { PLC } \\
\text { PLC/PLA2 }\end{array}$ & $\begin{array}{l}\uparrow \text { IP", }\left[\mathrm{Ca}^{2+}\right] \mathrm{i}, \mathrm{PGE} 2 \\
\uparrow \mathrm{IP},\left[\mathrm{Ca}^{2+}\right] \mathrm{i}, \downarrow \mathrm{AMPc} \\
\uparrow \mathrm{IP} 3, \text { ac arachidonique }\end{array}$ \\
\hline $\begin{array}{l}\text { Cellules nerveuses } \\
\text { - NG108-15 } \\
\text { - NCB-20 }\end{array}$ & $\begin{array}{l}\text { B2 } \\
\text { B2 }\end{array}$ & $\begin{array}{l}\text { Goq/PLC } \\
\text { PLC }\end{array}$ & $\begin{array}{l}\uparrow \stackrel{\mathrm{IP} 3}{ } \\
\uparrow\left[\mathrm{Ca}^{2+}\right] \mathrm{i}, \mathrm{AMPc}\end{array}$ \\
\hline $\begin{array}{l}\text { Mésangial } \\
\text { - rein (rat) }\end{array}$ & B1-B2 & PLC/PLA2 & $\begin{array}{l}\uparrow \mathrm{IP} 3,\left[\mathrm{Ca}^{2+}\right] \mathrm{i}, \mathrm{PGE} 2, \downarrow \mathrm{AMPc} \text {. } \\
\text { Prolifération, contraction }\end{array}$ \\
\hline $\begin{array}{l}\text { Endothélial } \\
\text { - artère pulmonaire (bovin) } \\
\text { - aorte (bovin) } \\
\text { - aorte (lapin) } \\
\text { - jugulaire (lapin) } \\
\text { - artère carotide (chien) }\end{array}$ & $\begin{array}{l}\text { B1-B2 } \\
\text { B1-B2 } \\
\text { B1 } \\
\text { B2 } \\
\text { B2 }\end{array}$ & $\begin{array}{l}\text { PLC/PLA2/NOSc } \\
\text { Goi, G } \alpha q \\
\text { PLA2/NOSc } \\
\text { PG indépendant } \\
\text { - }\end{array}$ & $\begin{array}{l}\uparrow I \mathrm{IP} 3,\left[\mathrm{Ca}^{2+}\right] \mathrm{i}, \mathrm{PGI} 2, \mathrm{PAF}, \mathrm{NO} \\
\uparrow \mathrm{PGI} 2, \mathrm{NO}, \mathrm{GMPc} \\
\text { contraction } \\
\text { contraction } \\
\text { relaxation via PGI2, EDHF et NO }\end{array}$ \\
\hline $\begin{array}{l}\text { Macrophage } \\
\text { - RAW264.7 }\end{array}$ & B1 & - & $\uparrow$ IL1, TNF libération \\
\hline $\begin{array}{l}\text { Muscle lisse } \\
\text { - aorte de rat }\end{array}$ & B1-B2 & PLC/PLA2 & $\uparrow \mathrm{IP3},\left[\mathrm{Ca}^{2+}\right] \mathrm{i}, 6$ kétoPGF1 $\alpha$ \\
\hline Ostéoblaste (rat) & B1-B2 & PLC/PLA2 & $\uparrow\left[\mathrm{Ca}^{2+}\right] \mathrm{i}, \mathrm{PGE} 2$ \\
\hline
\end{tabular}

Abréviations: PLC, phospholipase $C$; PLA2, phospholipase A2; IP3, inositol triphosphate; PG, prostaglandin; NOSc, nitric oxide synthase constitutive ; $P A F$, platelet activating factor; NO, monoxyde d'azote; $I L$, interleukine; EDHF, endothelium derived hyperpolarizing factor; $T N F$, tumor necrosis factor ; $\left[\mathrm{Ca}^{2+}\right] \mathrm{i}$ : concentration du calcium libre cytosolique.

$\mathrm{I}_{2}$ mais aussi les leukotriènes et le PAF (platelet activating factor) [15]. La libération des eicosanoïdes est une composante importante du mécanisme d'action de la BK puisque, dans la majorité des cas, l'inhibition de la synthèse des eicosanoïdes diminue fortement les effets de la BK [15]. La stimulation de la PLA2 peut être partiellement indépendante de l'activation préalable de la PLC dans les fibroblastes Swiss3T3, indiquant un couplage avec des protéines $\mathrm{G}$ différentes. De même, la $\mathrm{BK}$ induit la contraction des cellules mésangiales par deux mécanismes distincts, l'un faisant appel à la voie de la PLC et l'autre dépendant de la formation des prostaglandines [27]. En ce qui concerne les relations avec l'adénylyl cyclase, la BK est capable de stimuler indirectement la production d'AMPc dans les fibroblastes par l'intermédiaire des prostaglandines E2 agissant de façon autocrine [15]. Dans la cellule mésangiale, nous avons pu montrer que la BK inhibait la formation d'AMPc via l'activation de la PKC [28]. Par ailleurs, Boyajian et al. [29] ont rapporté que le $\left[\mathrm{Ca}^{2+}\right] \mathrm{i}$ mobilisé après stimulation des cellules NCB-20 par la BK inhibait directement l'adénylyl cyclase. Enfin, la BK stimule indirectement l'activité de la phospholipase D via la PKC [30] et active des tyrosine kinases capables de phosphoryler la protéine p21 ras GTPase activating protein (GAP) [31].
Bien que les mécanismes de transduction mis en jeu par l'activation du récepteur $\mathrm{B} 1$ soient moins bien connus, ils ne semblent finalement pas très différents de ceux du récepteur B2. La dés-Arg ${ }^{9}$-BK stimule la synthèse de prostaglandines par les cellules fibroblastiques, les cellules endothéliales et les ostéoblastes [15, 18]. La dés-Arg'-BK élève également la concentration du $\left[\mathrm{Ca}^{2+}\right] \mathrm{i}$ dans les ostéoblastes [18], les cellules mésangiales [17] et les cellules endothéliales provenant d'artère pulmonaire de bœuf [32]. Cependant, alors que l'activation du récepteur B2 mobilise préférentiellement le calcium à partir des réserves intracellulaires, l'activation du récepteur B1 semble asso- 


\section{RÉFÉRENCES}

25. Borkowski JA, Ransom RW, Seabrook GR, Trumbauer M, Chen H, Hill RG, Strader CD, Hess JF. Targeted disruption of a B2 bradykinin receptor gene in mice eliminates bradykinin action in smooth muscle and neurons. J Biol Chem 1995; 270 : 1370610

26. Liao JK, Homcy CI. The G proteins of

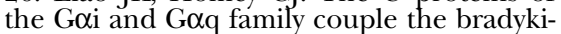
nin receptor to the release of endotheliumderived relaxing fator. J Clin Invest 1993; 92: 2168-72.

27. Bascands JL, Pecher C, Bompart G, Rakotoaryvony J, Leung-Tack J, Girolami JP. Bradykinin-induced in vitro contraction of rat mesangial cells via a B2 receptor type. Am J Physiol 1994; 267: F871-8.

28. Bascands JL, Pecher C, Girolami JP. Indirect inhibition by bradykinin of cyclic AMP generation in isolated rat glomeruli and cultured mesangial cells. Mol Pharmacol $1993 ; 44: 818-26$.

29. Boyajian CL, Garrtsen A, Cooper DMF. Bradykinin stimulates $\mathrm{Ca}^{2+}$ mobilization in NCB-20 cells leading to direct inhibition of adenylylcyclase. I Biol Chem 1991; 266: 8 : 4995-5003.

30. Pyne S, Pyne NJ. Bradykinin stimulates phospholipase D in primary cultures of guinea pig tracheal smooth muscle. Biochem Pharmacol 1993; 45: 593-603.

31. Leeb-Lumberg LMF, Song XH. Identification of p125, a component of a group of $120 \mathrm{kDa}$ proteins that are phosphorylated on tyrosine residues in response to bradykinin and bombesin stimulation, in antiras GTPase activating protein immunoprecipitates of Swiss 3T3 cells. J Biol Chem 1993; 268: 9945-8.

32. Smith JA, Webb C, Holford J, Burgess GM. Signal transduction pathways for $B_{1}$ and $\mathrm{B}_{2}$ bradykinin receptors in bovine pulmonary artery endothelial cells. Mol Pharmacol 1995 ; 47 : 525-34.

33. Mombouli JV, Vanhoute PM. Kinins and endothelial control of vascular smooth muscle. Annu Rev Pharmacol Toxicol 1995; $35: 679-705$.

34. Margolius HS. Kallikreins and Kinins: some unanswered questions about system characteristics and roles in human disease. Hypertension 1995; 26 : 221-9.

35. Bascands JL, Marin-Castano MA, Bompart G, Pecher C, Gaucher M, Girolami JP. Postnatal maturation of the kallikrein-kinin system: from enzyme activity to receptor gene expression. J Am Soc Nephrol 1996; 7 : ciée à un influx de calcium plus important qui est inhibé par le chlorure de nickel mais inaltéré par les antagonistes des canaux calciques de type L. L'identité de ces canaux calciques et le mécanisme déclenchant leur ouverture restent à déterminer.

\section{Le récepteur $\mathrm{B} 2$ de la cellule endothéliale}

Du fait de l'équipement enzymatique spécifique de la cellule endothéliale et en particulier de la présence de la forme constitutive de la synthase du monoxyde d'azote (NOSc), l'activation du récepteur B2 de la BK se traduit par des effets spécifiques qui sont fortement impliqués dans le contrôle de la vasomotricité [33]. En plus de la stimulation classique des

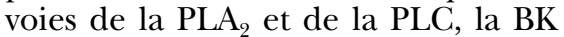
active la NOSc. Le monoxyde d'azote (NO) produit dans la cellule endothéliale, va diffuser et stimuler princi- palement la guanylyl cyclase soluble des cellules musculaires lisses (CML). Cette activation entraîne la formation du GMP cyclique responsable de la relaxation des CML (figure 3).

Récemment, la stimulation par la BK d'une nouvelle voie de transduction a été suggérée. Les auteurs ont montré que l'effet vasodilatateur de la BK persiste malgré le blocage de la production des prostaglandines et du monoxyde d'azote. Cet effet est attribué à un nouveau facteur endothélial [33], l'EDHF (endothelium-derived hyperpolarizing factor) (figure 3). La nature exacte de ce nouveau facteur diffusible demeure encore inconnue. L'EDHF entraîne une hyperpolarisation de la CML vasculaire et une activation des canaux potassiques. L'effet systémique vasodilateur bien connu de la BK nécessite donc l'association cellule endothéliale/cellule musculaire lisse. En revanche, dans certaines situations associées à une

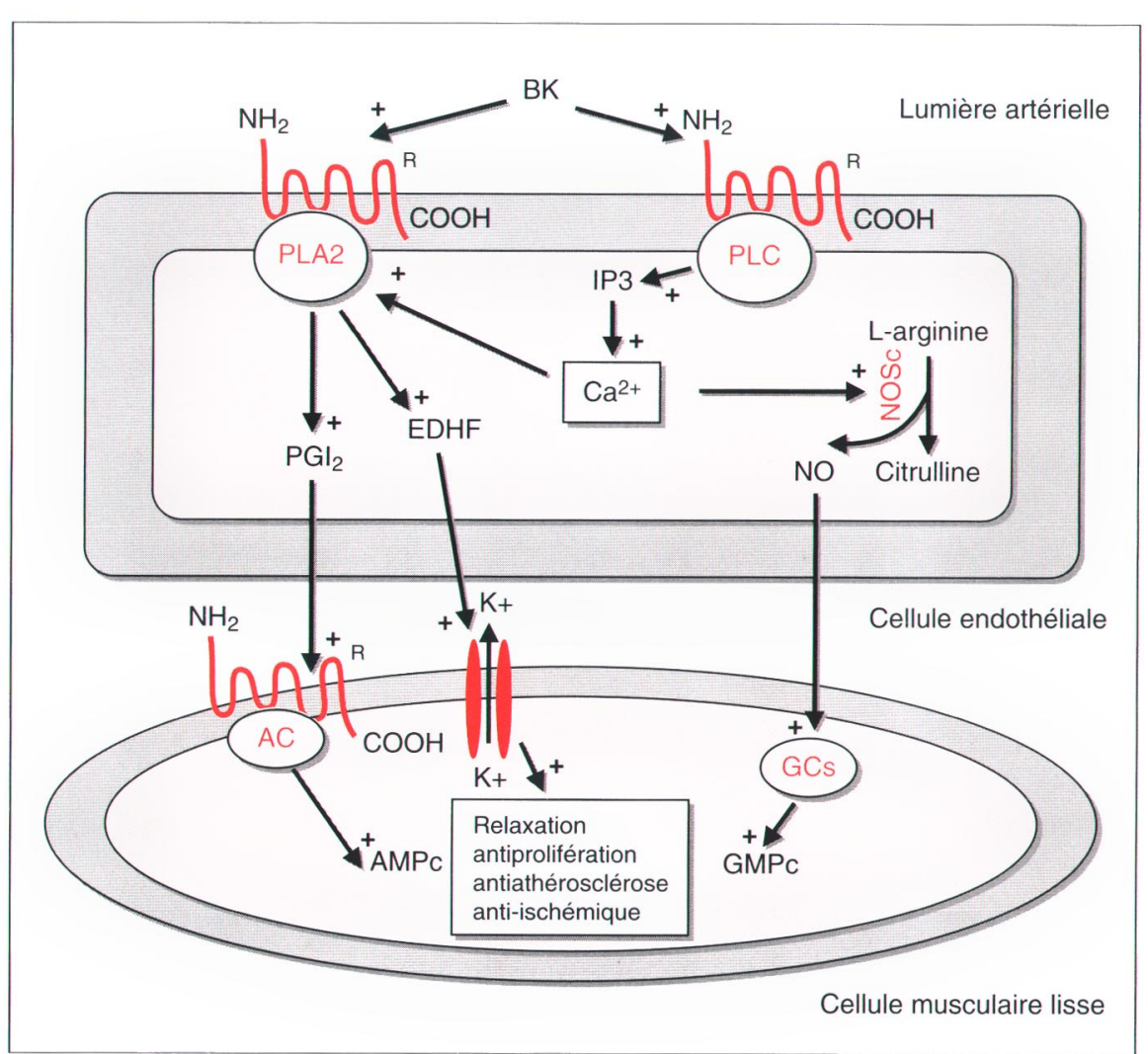

Figure 3. Le récepteur B2 de la cellule endothéliale. Transduction du signal permettant la relaxation de la cellule musculaire lisse. $B K$ (bradykinine), $R$ (récepteur), EDHF (endothelium-derived hyperpolarizing factor), PGI2 (prostaglandine 12), NOSc (NO synthase constitutive), GCs (guanylyl cyclase soluble), AC (adénylyl cyclase), PLA2 (phospholipase A2), PLC (phospholipase C). 
lésion ou à une absence d'endothélium, la BK, en agissant directement sur la CML, pourrait devenir un puissant et donc potentiellement «dangereux» vasoconstricteur susceptible d'aggraver ces situations.

\section{Mécanismes de régulation}

Les mécanismes de régulation doivent être envisagés à deux niveaux : la disponibilité de l'agoniste, d'une part, l'expression et l'activation du récepteur, d'autre part.

La demi-vie de la BK dans la circulation, est estimée à environ 15 secondes [7], et résulte de l'équilibre entre sa formation par la kallicréine et sa dégradation par les kininases. L'activité de la kallicréine tissulaire est stimulée par la déplétion sodée et par les minéralocorticoïdes comme en témoignent les nombreux travaux du groupe de H. Margolius [34]. Le contrôle de la dégradation de la BK reste, en revanche, un enjeu thérapeutique de premier plan. Ainsi, lors de l'utilisation thérapeutique des inhibiteurs de l'enzyme de conversion, l'élévation de la concentration de bradykinine serait impliquée dans certains effets secondaires (la toux en particulier) de cette classe de médicaments.

Les études portant sur la régulation de l'activation des récepteurs ont montré des mécanismes de désensibilisation analogues à ceux décrits pour d'autres récepteurs à sept domaines faisant intervenir des phénomènes d'internalisation [15]. Il ne semble pas y avoir de désensibilisation hétérologue entre l'activation du récepteur B1 et celle du récepteur B2 [17]. Toutefois, on observe dans les divers types cellulaires étudiés une désensibilisation homologue [15], à l'exception du récepteur B1 des cellules endothéliales d'artère pulmonaire de bœuf qui ne présente pas de phénomène de désensibilisation [32]. Le contrôle de l'expression du gène codant pour le récepteur $\mathrm{B} 2$ commence à peine à être étudié. Un premier travail montre que la synthèse de cette protéine dans le rein de rat augmente jusqu'à l'âge de 8 semaines avant de se stabiliser à un niveau inférieur [35].

In vitro, une augmentation de l'AMPc intracellulaire par la forskoline, la toxine cholérique ou la toxine per- tussique s'accompagne de l'augmentation du nombre des récepteurs B2, bloquée par l'actinomycine $\mathrm{D}$ ou le cycloheximide [15]. Quelques équipes ont montré que l'expression des oncogènes ras et $d b l$ augmente également la synthèse du récepteur B2; à l'inverse, la dexaméthasone diminue le nombre des récepteurs de la $\mathrm{BK}$ [15]. Des manœuvres plus physiologiques effectuées chez le rat ont montré que la déplétion sodée diminue le nombre des récepteurs B2 alors que la restriction hydrique et la surcharge en sel les augmentent [36]. Dans toutes ces expériences, aucune modification de l'affinité du récepteur n'a été mise en évidence. Le récepteur B1, peu détectable dans des situations physiologiques, est fortement induit dans des situations pathologiques de type inflammatoire, provoquées expérimentalement par le lipopolysaccharide (LPS) et l'interleukine $1 \beta$ (IL1 $\beta)$. Cette induction, spécifique du type B1, est abolie par des inhibiteurs de la transcription et de la traduction [15].

\section{Effets biologiques de la BK}

C'est principalement pour son effet vasodépresseur sur le système cardiovasculaire que la BK est connue. De nombreux travaux montrent que la perfusion de BK entraîne une forte diminution de la pression sanguine artérielle [37]. Cet effet pharmacologique est confirmé et renforcé par l'utilisation d'antagonistes qui bloquent l'activité tonique vasodépressive $d u$ récepteur de type $\mathrm{B} 2$. Le mécanisme d'action de cet effet de la BK est maintenant assez bien connu et repose sur la production, par la cellule endothéliale, de substances vasodilatatrices (prostaglandines, NO, EDHF) qui, par diffusion, vont relaxer la cellule musculaire lisse sous-jacente [33, 37].

L'effet bénéfique des inhibiteurs de l'enzyme de conversion, observé sur la correction de l'hypertrophie de la média vasculaire associée à certaines anomalies vasculaires, a suggéré que la BK pourrait avoir une action antiproliférative sur les cellules musculaires lisses vasculaires. Cet effet protecteur de l'intégrité de la paroi vasculaire semble indépendant de l'effet vasodépresseur [37]. Pour pré- ciser le mécanisme de cet effet protecteur de tissu, il été a montré que la BK active des voies métaboliques bénéfiques telles que la production d'ATP et de phosphocréatine tout en stimulant le transport de glucose. Ces effets de protection tissulaire sont également à rapprocher de l'effet protecteur de la BK contre l'ischémie myocardique [37]. En revanche, plusieurs travaux effectués sur des cellules en culture montrent que la BK a une action comitogène $[17,38]$ et peut entraîner une contraction des cellules musculaires lisses. Cette action contractile directe sur la monocouche endothéliale augmente la perméabilité des capillaires et explique le rôle de la BK dans la formation des odèmes [18].

$\mathrm{Au}$ niveau rénal, la perfusion de BK dans l'artère rénale entraîne une augmentation du débit sanguin rénal sans modification du débit de filtration glomérulaire. Un tel effet est expliqué par une contraction des cellules mésangiales rénales qui provoque une diminution du coefficient d'ultrafiltration glomérulaire [7, 27]. En ce qui concerne la localisation rénale des récepteurs de la $\mathrm{BK}$, seule la distribution de récepteurs de type $\mathrm{B} 2$ est connue [7]. En fonction de la répartition des sites B2 le long du néphron, on peut distinguer au moins deux types d'actions: l'une sur l'hémodynamique glomérulaire et la circulation médullaire et l'autre sur les transferts ioniques et hydriques. En effet, l'activation des récepteurs B2 présents au niveau glomérulaire et sur les microvaisseaux médullo-papillaires [7, 39] induit un tonus vasodilatateur nécessaire au maintien de l'hémodynamique glomérulaire et de la microcirculation médullaire. D'autre part, dans la partie distale du néphron, la stimulation des récepteurs B2 situés sur les tubules collecteurs inhibe la formation d'AMPc induite par l'hormone antidiurétique [40].

Bien que la BK ait été initialement connue pour ses effets vasodilatateurs, de nombreux autres effets biologiques de ce peptide dans d'autres tissus non vasculaires ont été rapportés (Tableau III).

Le rôle des kinines dans le système nerveux a fait l'objet d'une revue récente [41]. Au niveau périphérique la BK stimule les terminaisons nerveuses des fibres $\mathrm{C}$ impliquées dans 


\section{RÉFÉRENCES}

36. Emond C, Bascands JL, Cabos-Boutot G, Pecher C, Girolami JP. Effect of changes in sodium or water intake on glomerular B2-kinin binding sites. Am J Physiol 1989; 257 : F353-8.

37. Linz W, Wiemer G, Gholke P, Unger T, Schölkens BA. Contribution of kinins to the cardiovascular actions of angiotensin-converting enzyme inhibitors. Pharmacol Rev 1995; 47: 25-49.

38. Khiene K, Rozengurt E. Synergistic stimulation of DNA synthesis by bradykinin and vasopressin in Swiss 3T3 cells. J Cell Physiol $1994 ; 160$ : 502-10.

39. Roman RJ, Kaldunski ML, Scicli AG, Carretero OA. Influence of kinins and angiotensin II on the regulation of papillary blood flow. Am J Physiol 1988; 255: 24: F690-8.

40. Dixon BS, Breckon R, Fortune J, Sutherland E, Simon RF, Anderson RJ. Bradykinin activates protein kinase $\mathrm{C}$ in cultured rat cortical collecting tubular cells. Am J Physiol 1989; 257: F808-17.

41. Walkers K, Perkins M, Dray A. Kinins and kinin receptors in the nervous system. Neurochem Int 1995; 26 : 1-16.

42. Farmer SG. The pharmacology of bradykinin in human airways. In : Page CP, Metzger WJ, eds. Drugs and the lung. New York: Raven Press Ltd, 1994: 449-65.

43. Proud D, Kaplan AP. Kinin formation: mechanisms and role in inflammatory disorders. Annu Rev Immunol 1988; 6: 49-83.

44. Gohlke P, Linz W, Schoelkens BA, Kuwer I, Bertenbach S, Schnell A, Unger T. Angiotensin-converting enzyme inhibition improves cardiac function. Role of bradykinin. Hypertension 1994; 23 : 411-8.

45. Brew EC, Mitchell MB, Rehring TF, Gamboni-Robertson F, McIntyre JRRC, Harken AH, Banerjee A. Role of bradykinin in cardiac functional protection after global ischemia-reperfusion in rat heart. Am J Physiol 1995 ; 269 ; 38 : H1370-8.

46. Schuh JR, Blehm DJ, Frierdich GE, McMahon EG, Blaine EH. Differential effects of renin-angiotensin blockade on atherogenesis in cholesterol fed rabbits. J Clin Invest 1993; 91 : 1453-8.

47. Farhy RD, Ho KL, Carretero OA, Scicli AG. Kinins mediate the antiproliferative effect of ramipril in rat carotid artery. Biochem la transmission d'influx liée à la nociception. Au niveau du système nerveux central les récepteurs B2 de la BK ont été localisés dans plusieurs régions, cortex, hippocampe, hypothalamus, hypophyse, medulla oblongata, cervelet et moelle épinière [41]. La majorité des sites de liaison se trouvant sur les vaisseaux sanguins l'action de la BK repose essentiellement sur ses effets vasculaires (vasodilatation via les prostaglandines et le NO). L'injection intracérébro-ventriculaire de BK entraîne une élévation de la pression sanguine artérielle et une augmentation de la fréquence cardiaque par l'intermédiaire des récepteurs B2. Le rôle précis de la BK dans le contrôle central de la pression sanguine (chez l'animal normotendu) n'a pas été clairement démontré, toutefois des études d'autoradiographie ont permis de montrer la présence de récepteurs de la BK dans les régions du cerveau associées au contrôle central cardiovasculaire (noyaux du tractus solitaire, et noyau dorsal du vague). En revanche, chez le rat spontanément hypertendu, l'implication du récepteur B1 semble mieux établie puisque l'administration intracérébro-ventriculaire d'antagoniste B1 diminue la fréquence cardiaque et la pression sanguine artérielle chez ces animaux [41].

L'effet des kinines sur le système gastro-intestinal repose essentiellement sur son action motrice sur les muscles lisses via la libération de prostaglandines et de monoxyde d'azote. Au niveau des épithéliums, la BK stimule principalement la sécrétion de chlorure [7, 18].

$\mathrm{Au}$ niveau pulmonaire la BK entrâ̂ne, dans la plupart des espèces, une bronchoconstriction et une stimulation des nerfs sensoriels associées à une augmentation de la sécrétion de mucus [42]. Son implication probable dans les maladies inflammatoires des voies aériennes a suscité de premières investigations cliniques.

\section{Implication des récepteurs $B 1$ et $B 2$ en pathologie: effets bénéfiques ou délétères?}

Si de nombreux modèles expérimentaux suggèrent l'implication de la BK en pathologie, on dispose de peu de démonstrations véritables. On peut cependant distinguer deux grands types de situations opposées, celles associées à une augmentation de la production ou de la réactivité tissulaire pour la bradykinine (allergie, inflammation, œedème) et, à l'inverse, des états caractérisés par un déficit de production ou une diminution de l'efficacité du récepteur (anomalies vasculaires). Par ailleurs, la définition d'un rôle pathologique de la bradykinine est rendue encore plus complexe par le fait que ce peptide peut activer le récepteur B2 mais également le récepteur B1 dont l'expression peut être fortement induite par les cytokines. On est donc confronté à plusieurs questions: quels effets, bénéfiques ou délétères, peut-on attribuer à la bradykinine? quels récepteurs, B1 ou B2, sont impliqués dans ces effets?

- Allergies, inflammation, syndromes douloureux, affections associées à la présence d'œdèmes

A l'heure actuelle, on étudie la capacité des antagonistes des récepteurs B1 et B2 d'intervenir dans le contrôle de ces situations pathologiques. Chez l'homme, la BK inhalée produit une importante bronchoconstriction chez les asthmatiques mais pas chez les sujets sains [43]. Au niveau des voies aériennes supérieures la BK provoque les mêmes symptômes que ceux observés dans les rhinites induites par le froid (rhinovirus) ou de type allergique [43]. C'est donc dans ces situations qu'ont été effectuées les premières tentatives d'utilisation d'antagonistes des récepteurs $\mathrm{B} 2$ de la $\mathrm{BK}$. Si la première génération d'antagonistes B2 a été quelque peu décevante du fait de leur courte demi-vie in vivo, le HOE 140 semble être efficace dans le traitement de l'asthme et des rhinites allergiques (allergie aux acariens) [13].

Le CP-0127, antagoniste dimérique du récepteur B2, fait l'objet d'études cliniques dans des affections aussi diverses que la pneumonie provoquée par l'hantavirus, les traumatismes crâniens et le choc septique. Cet antagoniste réduit l'œedème crânien formé lors du traumatisme améliorant ainsi la morbidité [13]. Dans le choc septique, du fait de la disparition de l'enzyme de conversion de la 


\begin{tabular}{|c|c|c|}
\hline \multicolumn{3}{|c|}{$\begin{array}{c}\text { Tableau III } \\
\text { EFFETS BIOLOGIQUES DE LA BRADYKININE }\end{array}$} \\
\hline Organes/Tissus & Récepteurs & Effets biologiques \\
\hline $\begin{array}{l}\text { Système nerveux central } \\
\text { et périphérique }\end{array}$ & B1-B2 & $\begin{array}{l}\text { - } \uparrow \text { débit sanguin, PG et cytokines - stimulation fibres } \\
\text { A } \delta \text { et C } \\
\text { - régulation du système cardiovasculaire } \\
\text { (via la libération de NA et de sérotonine) }\end{array}$ \\
\hline Iris & B2 & - contraction \\
\hline Trachée (préparation précontractée) & B2 & - relaxation (via les PG) \\
\hline Poumons & B2 & $\begin{array}{l}\text { - Bronchoconstriction (via stimulation des fibres } \\
\text { nerveuses libérant l'Ach) } \\
\text { - } \uparrow \text { sécrétion de mucus }\end{array}$ \\
\hline $\begin{array}{l}\text { Cœur } \\
\text { Artères coronaires }\end{array}$ & $\begin{array}{l}\text { B2 } \\
\text { B2 }\end{array}$ & $\begin{array}{l}\text { - } \uparrow \text { fréquence et débit cardiaque } \\
\text { - dilatation (via NO et GMPc) }\end{array}$ \\
\hline Rein & B1-B2 & $\begin{array}{l}-\uparrow \text { débit sanguin rénal } \\
-\downarrow \text { résistance artériole afférente } \\
\text { - diurétique, natriurétique }\end{array}$ \\
\hline Glandes sudoripares & B2 & - transport de l'eau et des électrolytes \\
\hline $\begin{array}{l}\text { lléon (cellules musculaires } \\
\text { et épithéliales) }\end{array}$ & B2 & - contraction (via PG), sécrétion de $\mathrm{Cl}-$ \\
\hline Duodénum & B2-B1 & - relaxation (B2) puis contraction (B1) \\
\hline $\begin{array}{l}\text { Côlon (cellules musculaires } \\
\text { et épithéliales) }\end{array}$ & B1 & - contraction, sécrétion de $\mathrm{Cl}-$ \\
\hline Vésicule biliaire & B2 & - sécrétion de bicarbonate \\
\hline Utérus & B2 & - contraction \\
\hline Ovaire & B2 & - rupture du follicule \\
\hline Spermatozoïde & B2 & $-\uparrow$ mobilité \\
\hline Fibroblastes & $\mathrm{B} 2$ & - $\uparrow$ synthèse de collagène \\
\hline
\end{tabular}

$P G$, prostaglandine; fibre $A$, fibre adrénergique; fibre $C$, fibre afférente primaire non myélinisée; NA, noradrénaline; Ach, acétylcholine; NO: monoxyde d'azote.

circulation, la BK est hydrolysée principalement par la carboxypeptidase-N et engendre la dés-Arg ${ }^{9}$-BK, agoniste sélectif du récepteur B1. L'activation de ce dernier entraîne une vasodilatation qui explique en partie le collapsus observé lors du choc septique. Dans ce type d'affection, l'emploi d'hétérodimères antagonistes des récepteurs B1 et B2 (CP-0364) semble désormais d'un intérêt évident [13].
L'utilisation d'antagonistes B1 reste pour l'instant expérimentale; elle est efficace pour diminuer l'hyperalgie provoquée par l'administration d'adjuvant de Freund dans l'articulation du genou chez le rat [41].

- Anomalies vasculaires : hypertension, ischémie de reperfusion et athérosclérose

Dans certaines hypertensions dues à une hyperproduction de rénine, on observe une stimulation du système rénine-angiotensine vasoconstricteur. La stratégie thérapeutique la plus fréquente est d'inhiber ce système, en particulier par les IE C qui, d'un côté, empêchent la formation d'angiotensine II et, de l'autre, bloquent la dégradation de la BK. Cependant, l'hypothèse selon laquelle une diminution de la concentration en BK, considérée comme un vasodilatateur endogène, pourrait participer au 
développement de l'hypertension n'est pas accueilli avec un enthousiasme général [34, 37, 44]. Dans l'hypertension rénovasculaire expérimentale, associée à une activation du système rénine-angiotensine vasoconstricteur, la plupart des études concluent à l'absence d'effet de la BK dans le mécanisme d'action des inhibiteurs de l'enzyme de conversion. Ce résultat est toutefois parfaitement en accord avec la forte diminution de l'activité du système kallicréine-kinine dans ce type d'hypertension. En revanche, dans les hypertensions provoquées par une surcharge sodée ou par un excès de minéralocorticoïdes, l'implication de la BK dans les effets des IEC a été plusieurs fois suggérée [34]. Un dernier argument en faveur d'un rôle des kinines est l'efficacité des IEC dans les hypertensions dites à rénine normale. On peut, dans ce cas encore, s'interroger sur leur mécanisme d'action: inhibition de la formation d'angiotensine II (déjà basse) ou inhibition de la dégradation des kinines.

Dans les situations de reperfusion après ischémie, soit expérimentale après occlusion des coronaires chez le chien, soit chez l'homme après un infarctus du myocarde, on observe une augmentation de la concentration en kinines dans le sang des sinus coronaires [37]. La perfusion de bradykinine, préalablement à l'ischémie, induit un bon effet cardioprotecteur [45]. L'effet métabolique des kinines semble plus déterminant que l'effet vasodilatateur [37].

Dans l'athérosclérose, induite chez le lapin par un régime riche en cholestérol, le traitement par un IEC, l'énalapril, réduit très significativement le nombre de plaques d'athérome avec une efficacité supérieure aux antagonistes des récepteurs AT1 de l'angiotensine II [46]. Dans ce travail il est fortement suggéré que l'atténuation de l'athérosclérose par les IEC pourrait passer par l'action des kinines.
En effet, ces dernières, en stimulant la libération de prostacyclines et de NO, participeraient à la diminution de la progression de la plaque d'athérome. De façon un peu différente, après désendothélisation de l'artère carotide par la technique du ballonnet, un IEC tel que le ramipril, diminue la néoformation de l'intima de manière plus spectaculaire qu'un traitement par le losartan (antagoniste du récepteur AT1 de l'angiotensine II). En revanche l'effet du ramipril est inhibé si on traite simultanément avec l'antagoniste B2, le HOE 140, ou un inhibiteur de synthèse de $\mathrm{NO}$, le L-NAME [47].

\section{Conclusion et perspectives}

Il ressort de l'ensemble de ces données que la $\mathrm{BK}$, exerce des effets bénéfiques dans les maladies vasculaires (vasodilatation et effets protecteurs tissulaires). Dans ce cadre, il serait donc intéressant de pouvoir mieux protéger la BK de la dégradation et de développer des agonistes de type non peptidique, résistant à la dégradation, ou encore de maintenir l'expression du récepteur B2. En revanche, le développement de nouveaux antagonistes des récepteurs B2 ayant une durée de vie plus longue serait tout à fait bénéfique dans le traitement des affections à caractère inflammatoire. De même, les antagonistes du récepteur $\mathrm{B} 1$ seraient tout à fait opportuns dans le traitement des inflammations et douleurs chroniques. Enfin, il est important de souligner que l'utilisation de ces différents antagonistes devra tendre à bloquer l'action de la BK dans certaines affections, mais aussi à éviter le blocage des récepteurs vasculaires qui serait néfaste, tant en situation physiologique que pathologique. Une partie de ces données, essentiellement centrées sur l'implication du récepteur B2, risque cependant d'évoluer considérablement avec l'amélioration des connaissances sur le récepteur B1 dont l'étude commence à peine

\section{Summary}

Bradykinin

The physiological and pathological effects of the nonapeptide bradykinin (BK) are mediated via the activation of at least two types of bradykinin receptors termed $\mathrm{B} 1$ and B2. The B2 type is the most prevalent under physiological conditions and is preferentially activated by BK. The B1 type is selectively activated by des-Arg9-BK and seems to be greatly induced in some pathological states. Both B1 and B2 bradykinin receptors have had their cDNAs cloned and belong to the seven transmembrane coupled G-protein receptor family. BK receptor activation stimulates the transduction pathways leading to the generation of different second messengers $\left(\mathrm{Ca}^{2+}\right.$, prostaglandins, NO, cGMP). It is suggested that bradykinin receptors are involved in various different physiological and physiopathological mechanisms such as the control of blood pressure and cardiovascular functions, the inflammatory reaction (allergy, asthma, oedema). Therefore it appears that bradykinin receptors activation can be involved in beneficial and deleterious effects and thus the development of both long lasting agonists and antagonists are of therapeutical interest.

\section{TIRÉS À PART}

J.P. Girolami. 